\title{
Paediatric Wandering Spleens in Malawi
}

\section{E Broadis ${ }^{1}$, M K Banda ${ }^{2}$, EM Molyneux ${ }^{2}$,} E Borgstein ${ }^{1}$

1.Department of Surgery, College of Medicine, Blantyre

2.Department of Paediatrics, Queen Elizabeth Central Hospital, Blantyre

\section{Introduction}

We describe two paediatric cases in which the diagnosis of torsion of a wandering spleen was delayed. To the best of our knowledge, these are the first cases to be described in Malawi, and highlight the importance of accurate ultrasonography in the work up and differential diagnosis of children presenting with an abdominal mass in Sub-Saharan Africa.

\section{Case I}

An 11-year-old male presented with a two-month history of abdominal pain and wasting and a one-month history of abdominal distension. He was otherwise fit and well with no significant past medical or family history. He was on no medications and had no known allergies.

On examination he was wasted with a weight of 19 kilograms. His abdomen was moderately distended with a huge tender mass extending from the right upper quadrant to the right lower quadrant.

A Fine Needle Aspiration, Full Blood Count, Hepatitis B Serology, Stool and Urine Culture and Microscopy, Bone Marrow Aspiration and Ultrasonography of the abdomen were performed. Ultrasonography reported a large mass 10 X $20 \mathrm{~cm}$, with normal liver, pancreas and kidneys.

The working diagnosis was Burkitts Lymphoma and he was commenced on Cyclophosphamide.

Unfortunately the fine needle aspiration was inconclusive and he did not respond to chemotherapy. He was taken to theatre for a laparotomy where a diagnosis of torsion of a wandering spleen was made (See Figure 1). The splenic artery was thrombosed and a splenectomy was performed. The child made an uneventful postoperative recovery and was discharged home on monthly benzathine penicillin prophylaxis.

\section{Case II}

An eight year old girl was referred from a district hospital with a one year history of abdominal distension. On further questioning she admitted to recurrent episodes of abdominal pain that had prevented her from attending school. At the referring hospital she had been given quinine and a blood transfusion, as her haemoglobin was only $3.7 \mathrm{~g} / \mathrm{dL}$. She had no other significant past medical history or family history. She was on no other medications and had no known drug allergies.

On examination she had a firm tender mass in the right upper quadrant but no other organomegaly was noted. Ultrasonography reported a large central well delineated solid mass and normal liver, pancreas and kidneys. The spleen was reported as being difficult to visualise, possibly small.

A fine needle aspiration suggested a mixed inflammatory background and was suspicious of malignancy and a repeat specimen was requested.

The working diagnosis was Burkitts lymphoma and she was commenced on cyclophosphamide whilst undergoing further investigations.

She responded poorly to chemotherapy and a repeat ultrasonogram showed little change in the size of the mass and asplenia. She was taken to theatre with the suspicion of a torted wandering spleen and this was confirmed at laparotomy when a necrotic spleen was removed. She made an uneventful post operative recovery and was discharged home on monthly benzathine penicillin prophylaxis.

\section{Figure 1}

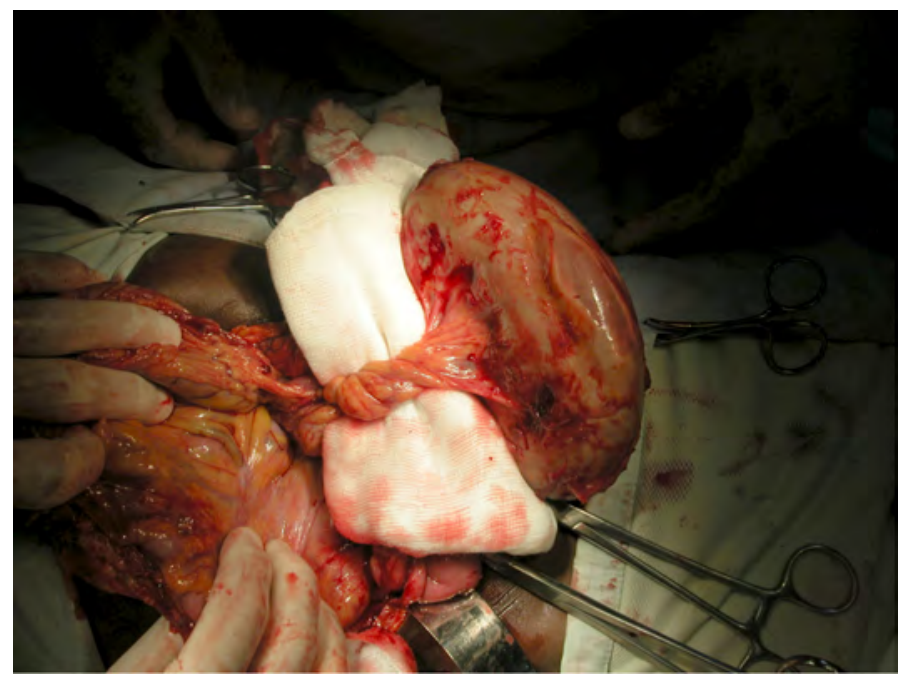

\section{Discussion}

The spleen is an intraperitoneal lymphoid organ normally located in the left hypochondrium. It is suspended by the gastrosplenic and splenorenal ligaments, which are formed by a double layer of peritoneum. The wandering spleen is the rare description of an abnormally positioned spleen, which is thought to occur due to laxity, abnormality or absence of the aforementioned ligaments. The wandering spleen is noted to have a longer than normal pedicle, and because of its intraperitoneal location, is able to move about the abdomen.

The incidence is unknown as many patients are asymptomatic, but based on a review of several large series of splenectomies, it is thought to be around $0.5 \%{ }^{1}$

Wandering spleen seems to have a preponderance to two main groups of patients: females of child bearing age, when it is postulated that hormonal changes in pregnancy result in laxity of the ligaments; and children, where there is a male to female ratio of around 6 to 1.2 There are less than 100 paediatric cases reported in the literature ${ }^{2}$.

Over half of cases present with an abdominal mass associated with intermittent abdominal pain. This makes the diagnosis difficult in Malawi where the predominant cause of an abdominal mass in children is Burkitts lymphoma, the prognosis of which is better with earlier commencement of chemotherapy. ${ }^{3}$ It is notable however, that spleens are often enlarged in Burkitts lymphoma and its absence should therefore raise doubts about the diagnosis.

Laboratory tests are often nonspecific but there are certain findings on radiology tests that should alert the clinician to the possibility of the diagnosis. On ultrasonography, the low position of the spleen or splenomegaly ${ }^{4}$ and on a contrast enhanced CT scan, the whorl sign is suggestive of torsion. ${ }^{5}$ The whorl sign is a circular, whorled structure in the splenic hilum which represents the torted, congested splenic pedicle. 
On reflection of our management of these cases, absence of the spleen in the left hypochondrium on ultrasound scan should have alerted us sooner to the possibility of a wandering spleen.

Splenic torsion has been reported to carry a mortality rate of up to $50 \%{ }^{6}$ due to infarction, rupture or abscess formation. In this situation, splenectomy is often inevitable. However, asplenia puts the patient at a high risk of post-splenectomy sepsis ${ }^{7}$ mainly from encapsulated bacteria but also malaria. For asymptomatic individuals with a wandering spleen many centres would now advocate splenopexy as the treatment of choice. Many surgical techniques are described for splenopexy including laparoscopic splenopexy, fixation of the capsule to the left upper quadrant, creation of a posterolateral extraperitoneal pocket at the level of the 12th rib, mobilisation of the splenic flexure with gastropexy, use of a polyglycolic mesh bag and hemisplenectomy with fixation of residual spleen. ${ }^{8-13}$

In our experience in Malawi, patients pose a further problem due to chronic malaria leading to enlarged spleens which have tough capsules that tear easily and are difficult to suture. This needs to be taken into consideration when planning the type of splenopexy.

\section{Conclusions}

Although rare, a torted wandering spleen should be included in the differential diagnosis of patients presenting with a tender abdominal mass. The spleen should always be looked for during the abdominal ultrasound examination, and its absence in the left upper quadrant should alert the clinician to the possibility of the diagnosis. Burkitts Lymphoma is often associated with splenomegaly, therefore the absence of a spleen should raise the possibility that the diagnosis is not Burkitts Lymphoma. If there is suspicion of splenic torsion on ultrasonography then a CT scan may confirm the diagnosis.

\section{References}

1. Kinori I, Rifkin MD. A truly wandering spleen. J Ultrasound Med 1988; 7: 101-5

2. Allen KB, Andrews G. Pediatric wandering spleen - the case for splenopexy: review of 35 reported cases in the literature. J Paed Surg 1989; 24(5): 432-5

3. Magrath I, Jack Lee Y, Anderson T et al. Prognostic factors in Burkitt's lymphoma importance of total tumor burden. Cancer 2006; 45(6): 1507-1515

4. Karmazyn B, Steinberg R, Gayer G et al. Wandering spleen - the challenge of ultrasound diagnosis. J Clin Ultrasound 2005; 33(9): 433-8

5.Thambidorai CR, Imtiaz A, Nafiqudin N. Torsion of wandering spleen with whorled appearance of the splenic hilum in CT scan. Med J Malaysia 2005; 60(5): 653-4

6. Moran JC, Shah U, Singer JA et al. Spontaneous rupture of wandering spleen: Case and review. Curr Surg 2003; 60: 310-2

7. Okabayashi T, Hanazaki K. Overwhelming post splenectomy infection syndrome review. World J Gastroenterol 2008; 14(2): 176179

8. Singla SL, Rattan KN, Sharma S et al. Torsion in a paediatric wandering spleen: case report and review. J Indian Assoc Pediatr Surg 2007; 12(1): 30-31

9. Esposito C, Settimi A, Centonze A et al. Hemisplenectomy and fixation of residual spleen. Pediatr Surg Int 2005; 21(6): 488-90

10. Rescorla FJ, West KW, Engum SA, Grosfeld JL. Laparoscopic splenic procedures in children: experience in 231 children. Ann Surg 2007; 246(4): 683-7

11. Fukuzawa H, Urushihara N, Ogura K et al. Laparoscopic splenopexy for wandering spleen: extraperitoneal pocket splenopexy. Pediatr Surg Int 2006; 22(11): 931-4

12. Soleimani M, Mehrabi A, Kashfi A et al. Surgical treatment of patients with wandering spleen: report of six cases with a review of the literature. Surg Today 2007; 37(3): 261-9

13. Palanivelu C, Rangarajan M, Senthilkumar R et al. Laparoscopic mesh splenopexy (sandwich technique) for wandering spleen. JSLS 2007; 11(2): 246-51 